\title{
Deformation analysis with Total Least Squares
}

\author{
M. Acar, M. T. Özlüdemir, O. Akyilmaz, R. N. Çelik, and T. Ayan \\ Istanbul Technical University, Division of Geodesy, Istanbul, Turkey \\ Received: 3 March 2006 - Revised: 13 July 2006 - Accepted: 13 July 2006 - Published: 25 July 2006
}

\begin{abstract}
Deformation analysis is one of the main research fields in geodesy. Deformation analysis process comprises measurement and analysis phases. Measurements can be collected using several techniques. The output of the evaluation of the measurements is mainly point positions. In the deformation analysis phase, the coordinate changes in the point positions are investigated. Several models or approaches can be employed for the analysis. One approach is based on a Helmert or similarity coordinate transformation where the displacements and the respective covariance matrix are transformed into a unique datum. Traditionally a Least Squares (LS) technique is used for the transformation procedure. Another approach that could be introduced as an alternative methodology is the Total Least Squares (TLS) that is considerably a new approach in geodetic applications. In this study, in order to determine point displacements, 3-D coordinate transformations based on the Helmert transformation model were carried out individually by the Least Squares (LS) and the Total Least Squares (TLS), respectively. The data used in this study was collected by GPS technique in a landslide area located nearby Istanbul. The results obtained from these two approaches have been compared.
\end{abstract}

\section{Introduction}

The history of geodetic deformation monitoring dates back to early 20th century. Technological advances and the improvements in the measurement techniques have made deformation monitoring a primary task in geodetic applications. Today space based positioning technology, and in particular the GPS offers an efficient monitoring methodology for areas at high risk, such as landslide phenomena, subsidence and structural deformation.

Deformation analysis methods have also been improved with the introduction of high capacity computers that enable

Correspondence to: M. T. Özlüdemir

(tozlu@itu.edu.tr) us to employ complex mathematical algorithms. For the deformation analysis several methods could be used. One of these methods is based on a Helmert or similarity coordinate transformation where the displacements and the regarding covariance matrix are transformed into a unique datum.

In general, the transforming data from one reference frame to another is solved by applying a coordinate transformation. Coordinate transformation is achieved by known coordinates of identical points in both coordinate systems. Although coordinate transformations are straightforward mathematically, they may cause several problems when applied, for various reasons, such as poor knowledge of the distortions and inconsistencies of the local datum, or even lack of sufficient knowledge of geodesy of people who use such transformations (Mitsakaki, 2004).

In classical approach, transformation parameters are estimated by the LS adjustment of the observation equations where only the observations are considered as stochastic. However, in some cases, design matrix elements also contain errors. Usually, this is ignored in classical least squares and this ignorance remains as an uncertainty in the solution results. TLS is a new method of parameter estimation in linear models that include error in -some or all- variables (EIV). Using TLS, coordinates of points in two coordinate systems are considered with their error component leading to the errors in the design matrix elements of the Helmert transformation equations. By this means, uncertainty of the estimated transformation parameters is reduced.

In this study, coordinate transformation which is one of these previously mentioned problems is examined. Displacements were determined by applying the transformation parameters obtained both from LS and TLS solutions. Obtained displacements were then tested and given in tabular form.

The geodetic deformation measurements used in this study were collected in Gürpinar landslide area nearby Istanbul. Since several landslides have taken place in Turkey causing severe property and life losses, geoscientists have been investigating the characteristics of landslide phenomenon. One of such studies is a geodetic deformation analysis project

Published by Copernicus GmbH on behalf of the European Geosciences Union. 
conducted in Gürpinar region. In this project a geodetic monitoring network covering the entire landslide area has been measured using GPS technique.

\section{3-D Helmert (similarity) transformation}

The three-dimensional conformal coordinate transformation is also known as the seven-parameter similarity transformation. It transfers points from one three-dimensional coordinate system to another (Wolf and Ghilani, 1997). The parameters are three translations (shift of origin), three rotations, and one parameter modelling a possible scale difference. The transformation preserves the shape of objects. The following formula has been used for the estimation of the parameters:

$$
\left[\begin{array}{l}
X \\
Y \\
Z
\end{array}\right]_{j}=\left[\begin{array}{l}
T_{x} \\
T_{y} \\
T_{z}
\end{array}\right]+(1+\lambda)\left[\begin{array}{ccc}
1 & -R_{z} & R_{y} \\
R_{z} & 1 & -R_{x} \\
-R_{y} & R_{x} & 1
\end{array}\right]\left[\begin{array}{l}
x \\
y \\
z
\end{array}\right]_{i}
$$

where $(1+\lambda)$ is scale factor, $R_{x}, R_{y}, R_{z}$ are rotation components. The translation terms $T_{x}, T_{y}$, and $T_{z}$ are the coordinates of the origin of the 3-D network.

The sub-matrices $A_{i}$ of the design matrix $A$ of LS observation equations for the estimation of the transformation parameters vector, $d k=\left\|\bar{T}_{x} \bar{T}_{y} \bar{T}_{z}(1+\lambda) R_{x} R_{y} R_{z}\right\|$ are in the following form.

$A_{i}=\left\|\begin{array}{ccccccc}1 & 0 & 0 & x_{i} & 0 & -z_{i} & y_{i} \\ 0 & 1 & 0 & y_{i} & z_{i} & 0 & -x_{i} \\ 0 & 0 & 1 & z_{i} & -y_{i} & x_{i} & 0\end{array}\right\| i=1, \ldots, n$

with $n$ being the number of the identical points of the transformation problem. The linear observation equations considering the relative coordinates according to the center of mass of the coordinate systems can then be written as follow,

$e=A \cdot d k-\ell$

$A=\left\|\begin{array}{ccccccc}1 & 0 & 0 & \Delta x_{1} & 0 & -\Delta z_{1} & \Delta y_{1} \\ 0 & 1 & 0 & \Delta y_{1} & \Delta z_{1} & 0 & -\Delta x_{1} \\ 0 & 0 & 1 & \Delta z_{1} & -\Delta y_{1} & \Delta x_{1} & 0 \\ 1 & 0 & 0 & \Delta x_{2} & 0 & -\Delta z_{2} & \Delta y_{2} \\ \vdots & \vdots & \vdots & \vdots & \vdots & \vdots & \vdots\end{array}\right\|$

$-\ell=\left[x_{s}-X_{1} y_{s}-Y_{1} z_{s}-Z_{1}\right]^{T}$ is the observation vector of the estimation problem. The following expressions are derived from Eqs. (3) and (4),

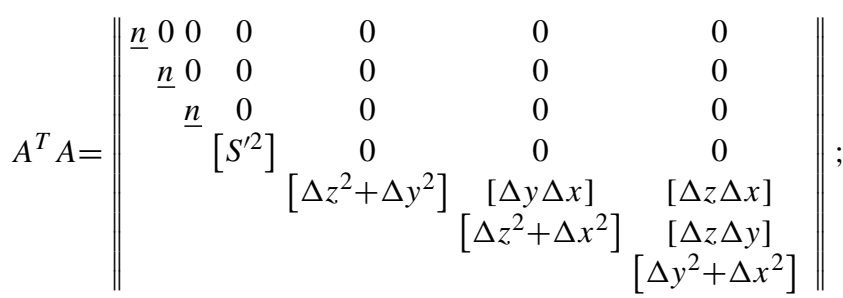

$$
-A^{T} \ell=\left\|\begin{array}{c}
-n X_{S} \\
-n Y_{s} \\
-n Z_{s} \\
{\left[\Delta x^{2}-\Delta X^{2}\right]} \\
{[-\Delta z \Delta Y+\Delta y \Delta Z]} \\
{[\Delta z \Delta X-\Delta x \Delta Z]} \\
{[\Delta x \Delta Y-\Delta y \Delta Y]}
\end{array}\right\|
$$

where $X_{s}, Y_{s}, Z_{s}$ are the coordinates of the center of the identical points in the first coordinate system.

$$
\begin{array}{ll}
S_{i}^{2}=\Delta X_{i}^{2}+\Delta Y_{i}^{2}+\Delta Z_{j}^{2} & S_{i}^{\prime 2}=\Delta x_{i}^{2}+\Delta y_{i}^{2}+\Delta z_{i}^{2} \\
\Delta X_{j}=X_{j}-X_{S} & \Delta x_{i}=x_{i}-x_{s} \\
\Delta Y_{j}=Y_{j}-Y_{S} & \Delta y_{i}=y_{i}-y_{s} \\
\Delta Z_{j}=Z j-Z_{s} & \Delta z_{i}=z_{i}-z_{s} \\
X_{S}=\sum X_{j} / n & x_{S}=\sum x_{i} / n \\
Y_{S}=\sum Y_{j} / n & y_{S}=\sum y_{i} / n \\
Z_{S}=\sum Z_{j} / n & z_{S}=\sum z_{i} / n
\end{array}
$$

After transformation parameters are estimated by LS, $x_{i}, y_{i}, z_{i}$ coordinates are transformed to $X_{j}, Y_{j}, Z_{j}$ coordinate system (Ayan, 1981; Ayan, 2001).

\section{Total Least Squares}

TLS estimation method has been introduced by Golub and Van Loan (1980) and had been an alternative to the classical LS method for the problems where both the observations and the elements of the design matrix are erroneous. The mathematical model of the classical TLS that is identical to the generalised LS method is as follows

$\ell+e_{\ell}=\left(A-E_{A}\right) \cdot d k(\operatorname{rank}(A)=m<n)$

where $e_{\ell}$ and $E_{A}$ are the error vector of observations and the error matrix of the design matrix $A$, both assumed to have independently and identically distributed rows with zero mean and the same variance.

The system given in Eq. (7) does in fact represent a nonlinear Gauss-Helmert $(\mathrm{GH})$ model and cannot be solved easily by standard linearisation (Lenzmann and Lenzmann, 2004). The solution of such a non-linear GH model depends on the extended linearised equations (using Taylor expansion) including the condition equations with unknowns so that the system will have both random and fixed unknowns (Koch, 1999). However, since the original system of equations is non-linear, the estimation of the unknowns is obtained within an iterative procedure where the iteration runs until the differences between the consecutive estimation of both random and fixed unknowns are in a predefined range. The disadvantage of such a solution is that it does not guarantee the convergence to the true solution of Eq. (7) and is rarely favourable (Mikhail and Ackermann, 1976). Especially, in our application, namely, the coordinate transformation, the distribution of the identical points may increase the 
possibility of achieving such a misleading result. In the following, we propose a more straight forward method, TLS, which uses a direct solution and guarantees the true solution of Eq. (7) which we call EIV model.

The TLS method performs an optimisation, minimising the error elements in Eq. (7) such that (Markovsky et al., 2004; Golub and Van Loan, 1980, Van Huffel and Vandewalle, 1991),

$\min _{\left[e_{\ell} ; E_{A}\right]}\left\|\left[E_{A} ; e_{\ell}\right]\right\|_{F}$

subject to $: \ell+e_{\ell}=\left(A-E_{A}\right) \cdot d k$

where $\|H\|_{F}$ denotes the Frobenius norm of an $n \times m$ matrix $H$, which is defined by

$$
\|H\|_{F}=\sqrt{\sum_{i=1}^{n} \sum_{j=1}^{m} h_{i j}^{2}}=\sqrt{\operatorname{tr}\left(H^{T} H\right)}
$$

where $t r$ denotes the trace operation and $\left[E_{A} ; e_{\ell}\right]$ denotes the $n \times(m+1)$ augmented error matrix constructed by adding vector of observation errors $\mathrm{e}_{l}$ to the error matrix $E_{A}$ as a right most column (Leon, 2002; Felus, 2004).

Formulation of the classical TLS solution of an estimation problem is given in detail by Akyilmaz (2005). The classical approach assumes that error vector and the design matrix elements have the same variance and uncorrelated.

In many applications the Singular Value Decomposition (SVD) is necessary to either determine the rank of a matrix or to determine whether the matrix is deficient in rank. Any matrix $A$ can be represented by the SVD as follows;

$A=U \Sigma V^{T}$

where $U=\left[u_{1,1}, \ldots, u_{1, n}, \ldots u_{n, 1}, \ldots, u_{n, n}\right] \in R^{n \times n}, \quad V=$ $\left[v_{1,1}, \ldots v_{1, m}, v_{1, m+1}, \ldots v_{m, m+1}, v_{m+1,1}, \ldots, v_{m+1, m+1}\right]$ $\in R^{(m+1) \times(m+1)}$ and $\sum=\left[\sigma_{1,1}, \ldots, \sigma_{1, m+1}, \ldots \sigma_{m+1, m+1}\right.$ $\left., \ldots, \sigma_{n, 1}, \ldots, \sigma_{n, m+1}\right] \in R^{n \times(m+1)}$ matrix with diagonal elements equal to the singular values and off-diagonal elements equal to zero. For simplicity, hereafter, we will use diagonal elements of matrix $\Sigma$ as $\Sigma=\operatorname{diag}\left(\sigma_{1}, \ldots, \sigma_{m}, \sigma_{m+1}\right)$ (Akyilmaz, 2005).

In practice, problems contain errors both in observation vector and the design matrix elements in a column-wise sense. Moreover, not all but some of the column elements of the design matrix are erroneous, that is the design matrix may contain error free columns. One example for such a problem is the coordinate transformation. Such a problem is known as Generalized TLS (GTLS) (Van Huffel, 1991).

Let $D$ be the $n \times n$ diagonal weight matrix of the observation equations. Further on, let $C$ be an $\left(m_{2}+1\right) \times\left(m_{2}+1\right)$ diagonal weight matrix that reflects the relative accuracies of the observations with respect to the design matrix elements in the columns of $A_{2}$ which occupies the erroneous elements of the design matrix. By using matrix $C$, one can assign more weight to observations with respect to the columns of $A_{2}$ or vice versa. Regarding these definitions, mathematical model of GTLS can be written as follows (Van Huffel, 1991).

$\min _{\left[e_{\ell} ; E_{A 2}\right]}\left\|D \cdot\left[E_{A 2} ; e_{\ell}\right] \cdot C\right\|_{F}$

subject to $: \ell+\mathrm{e}_{\ell}=\left[\mathrm{A}_{1} ; \mathrm{A}_{2}+\mathrm{E}_{\mathrm{A} 2}\right] \cdot\left[\begin{array}{l}d \hat{k}_{1} \\ d \hat{k}_{2}\end{array}\right]$

Even though Van Huffel (1991) has given a complicated formulation for the solution of GTLS problem which contains full covariance matrix for the observation vector and the elements of matrix $A_{2}$, in geodetic applications, the covariance matrices are generally block-diagonal dominant matrices and using this property we can reduce the problem to the GTLS problem with uncorrelated data without loosing reasonable accuracy for the estimates of the unknown parameters. For the readers interested in GTLS solution of cross-correlated observations, we refer to Mühlich and Mester (2004) and Akyilmaz (2005).

Considering the diagonal weights for both observations and the elements of matrix $A_{2}$, the GTLS solution of the problem consist of the following three steps.

1) The QR factorisation of the augmented matrix $D$. $\left[A_{1} ; A_{2} ; \ell\right]$ is calculated such that

$Q^{T} \cdot D \cdot\left[A_{1} ; A_{2} ; \ell\right]=\left[\begin{array}{ccc}R_{11} & R_{12} & R_{1 b} \\ 0 & R_{22} & R_{2 b}\end{array}\right]$

2) Using the second row of the Eq. (12), the classical TLS solution $d \hat{k}_{2}$ for the reduced system

$\left[R_{22} ; R_{2 b}\right] \cdot C \cdot\left(C^{-1} \cdot\left[\begin{array}{c}d \hat{k}_{2} \\ -\ell\end{array}\right]\right) \approx 0$

is again obtained using the SVD of $\left[R_{22} ; R_{2 b}\right] \cdot \mathrm{C}=\mathrm{U} \cdot \Sigma \cdot \mathrm{V}^{T}$ and $d \hat{k}_{2}$ is estimated by

$$
\begin{array}{r}
d \hat{k}_{2}=-\frac{1}{c_{m_{2}+1} \cdot v_{m_{2}+1, m_{2}+1}} \\
\cdot C_{1 \ldots m_{2}} \cdot\left[v_{1, m_{2}+1}, v_{2, m_{2}+1}, \ldots, v_{m_{2}, m_{2}+1}\right]^{T}
\end{array}
$$

where $C_{1 \ldots m_{2}}=\operatorname{diag}\left(c_{1}, c_{2}, \ldots, c_{m_{2}}\right)$, i.e., the diagonal elements of the matrix $C$ in the first $m_{2}$ rows (or columns). $c_{m_{2}+1}$ is the lower-most diagonal element of the matrix $C$ that is the weight of observation vector with respect to the columns of $A_{2}$.

3) The parameters $d \hat{k}_{1}$ are then solved by using the first row of the Eq. (12) through a back-substitution of the parameters $d \hat{k}_{2}$ estimated in the second step as,

$R_{11} \cdot d \hat{k}_{1}=R_{1 b}-R_{12} \cdot d \hat{k}_{2}$

or in a more familiar form

$d \hat{k}_{1}=R_{11}^{-1} \cdot\left(R_{1 b}-R_{12} \cdot d \hat{k}_{2}\right)$ 


\section{Deformation analysis using similarity transformation}

Datum consistency between the point coordinates computed from different observation campaigns can be accomplished by employing the similarity transformation. Moreover, the moving points can also be determined by the application of similarity transformation consecutively. In fact, similarity transformation is employed for transition from one datum into another without performing another adjustment. In other words, through similarity transformation the unknown parameters and their cofactor parameters are transformed from the current datum into a new datum (Caspary, 1987).

In this study the moving points have been determined using similarity transformation. This is done following the identification of the stable points in the network. The general analysis approach is described below.

Let the deformation vector and its magnitude for point $\mathrm{p}$ be

$$
\begin{aligned}
& d=\left[\begin{array}{c}
x_{\mathrm{k}}^{\mathrm{j}}-x_{\mathrm{k}}^{\mathrm{i}} \\
y_{\mathrm{k}}^{\mathrm{j}}-y_{\mathrm{k}}^{\mathrm{i}} \\
z_{\mathrm{k}}^{\mathrm{j}}-z_{\mathrm{k}}^{\mathrm{i}}
\end{array}\right]=\left[\begin{array}{c}
d_{\mathrm{x}} \\
d_{\mathrm{y}} \\
d_{\mathrm{z}}
\end{array}\right] \\
& \|d\|=\sqrt{d^{\mathrm{T}} d} .
\end{aligned}
$$

To determine the significance of these deformation vectors, which are computed according to Eqs. (17) and (18), the $H_{0}$ null hypothesis reads

$H_{0}: d=0$

whereas the test value is computed as follows.

$T=\frac{d^{\mathrm{T}} Q_{d d}^{-1} d}{3 s_{0}^{2}}$

The parameter $s_{0}^{2}$ is given as follows:

$s_{0}^{2}=\frac{f_{\mathrm{i}} s_{0 \mathrm{i}}^{2}+f_{\mathrm{j}} s_{0 \mathrm{j}}^{2}}{f_{\mathrm{i}}+f_{\mathrm{j}}}$

where $s_{0}^{2}$ is common variance, $s_{0 \mathrm{i}}^{2}$ and $s_{0 \mathrm{j}}^{2}$ variance factors, and $f_{\mathrm{i}}$ and $f_{\mathrm{j}}$ are the degrees of freedom of each of the two observation campaigns, respectively. If the test quantity exceeds the critical value, $\mathrm{F}_{3, \mathrm{f} ; 1-\alpha}$, then the point displacement is proven statistically (Denli, 2004).

\section{Application of TLS to a landslide deformation}

As aforementioned, the numerical application of LS and TLS based solutions have been carried out on a GPS data set collected in a landslide area located in the periphery of Istanbul. In the landslide area, a monitoring network consisting of 13 points was established. The control points were established in stable areas out of the landslide region. The locations of the deformation points were determined according to the geotechnical investigations in the landslide region. GPS measurements were carried out in four periods between July 1996 and March 1998. In this study, the measurements of the periods October 1997 and March 1998 have been evaluated. The measurements in each period have been adjusted through free network adjustment procedure and their adjusted values and variance-covariance matrix have been computed.

After that, Helmert coordinate transformations were performed between two epochs by LS and TLS respectively. The identical points of the performed transformation are given in Table 1.

GTLS estimation can be formulated using the following matrix and vector partitioning:

$A=\left[A_{1} ; A_{2}\right]$ with

$A_{1}=\left|\begin{array}{ccc}1 & 0 & 0 \\ 0 & 1 & 0 \\ 0 & 0 & 1 \\ \vdots & \vdots & \vdots \\ \vdots & \vdots & \vdots \\ 1 & 0 & 0 \\ 0 & 1 & 0 \\ 0 & 0 & 1\end{array}\right|_{3 n \times 3} \quad ; A_{2}=\left|\begin{array}{cccc}\Delta x_{1} & 0 & -\Delta z_{1} & \Delta y_{1} \\ \Delta y_{1} & \Delta z_{1} & 0 & -\Delta x_{1} \\ \Delta z_{1} & -\Delta y_{1} & \Delta x_{1} & 0 \\ \vdots & \vdots & \vdots & \vdots \\ \vdots & \vdots & \vdots & \vdots \\ \Delta x_{n} & 0 & -\Delta z_{n} & \Delta y_{n} \\ \Delta y_{n} & \Delta z_{n} & 0 & -\Delta x_{n} \\ \Delta z_{n} & -\Delta y_{n} & \Delta x_{n} & 0\end{array}\right|_{3 n \times 3}$

$\beta_{1}=\left|\begin{array}{c}T_{x} \\ T_{y} \\ T_{z}\end{array}\right| ; \beta_{2}=\left|\begin{array}{c}(1+\lambda) \\ R_{x} \\ R_{y} \\ R_{z}\end{array}\right|$

and observation vector $\ell$ is

$\ell=\left|\begin{array}{ccc}X_{1}-x_{s} & Y_{1}-y_{s} Z_{1}-z_{s} & \cdots \\ \cdots & X_{n}-x_{s} Y_{n}-y_{s} & Z_{n}-z_{s}\end{array}\right|$

GTLS solution given through the Eq. (12) to Eq. (16) was applied in Eqs. (22) and (23). The given covariance matrices D and $\mathrm{C}$ of the Eqs. (12)-(16) are as follows (Akyilmaz, 2005):

$$
\begin{gathered}
\operatorname{diag}(\mathrm{D})=\left[\begin{array}{cccc}
\sum_{X_{1} X_{1}} & \sum_{Y_{1} Y_{1}} & \sum_{Z_{1} Z_{1}} & \\
\cdots & \sum_{X_{n} X_{n}} & \sum_{Y_{n} Y_{n}} & \sum_{Z_{n} Z_{n}}
\end{array}\right] \\
C=\left[\begin{array}{ccccc}
\frac{\operatorname{tr}\left(\sum_{x y z}\right)}{\operatorname{tr}(D)} & 0 & 0 & 0 & 0 \\
& \frac{\operatorname{tr}\left(\sum_{y z}\right)}{\operatorname{tr}(D)} & 0 & 0 & 0 \\
& & \frac{\operatorname{tr}\left(\sum_{x z}\right)}{\operatorname{tr}(D)} & 0 & 0 \\
& & & \frac{\operatorname{tr}\left(\sum_{x y}\right)}{\operatorname{tr}(D)} & 0 \\
& & & 1
\end{array}\right]
\end{gathered}
$$

where $\operatorname{tr}(\cdot)$ is the trace operator of the argument matrix, the elements of the matrix $D$ are the covariances of the observations (coordinates of the system X-Y-Z), $\sum_{x y z}, \sum_{y z}, \sum_{x z}, \sum_{x y}$ are the full and submatrices of the covariances of the identical points in the transformed coordinate system (system $x-y-z)$, respectively.

The estimated transformation parameters from both methods are summarised in Table 2.

Note the difference between the estimated values of LS and GTLS approaches. This difference mainly comes from 
Table 1. Coordinates of the identical points.

\begin{tabular}{lllllll}
\hline Point ID & \multicolumn{2}{l}{ Coordinates of first system } & \multicolumn{3}{l}{ Coordinates of second system } \\
\hline & $\mathrm{x}(\mathrm{m})$ & $\mathrm{y}(\mathrm{m})$ & $\mathrm{z}(\mathrm{m})$ & $\mathrm{X}(\mathrm{m})$ & $\mathrm{Y}(\mathrm{m})$ & $\mathrm{Z}(\mathrm{m})$ \\
\hline 3 & 4233187.8344 & 2308228.6785 & 4161469.1229 & 4233187.8612 & 2308228.7042 & 4161469.1383 \\
185 & 4233190.6059 & 2308518.3249 & 4161336.2582 & 4233190.6124 & 2308518.3166 & 4161336.2682 \\
2796 & 4233429.1004 & 2307875.2240 & 4161292.4034 & 4233429.1008 & 2307875.2239 & 4161292.4029 \\
2996 & 4233259.8205 & 2307712.3025 & 4161553.4880 & 4233259.8309 & 2307712.2990 & 4161553.5007 \\
5005 & 4233770.4580 & 2308340.5240 & 4160740.3286 & 4233770.4534 & 2308340.5219 & 4160740.3181 \\
\hline
\end{tabular}

Table 2. Transformation parameters from LS and GTLS solution.

\begin{tabular}{lll}
\hline Method & LS & GTLS \\
\hline $\mathrm{T}_{x}(\mathrm{~m})$ & $-199,86044569$ & $-49,65230024$ \\
$\mathrm{~T}_{y}(\mathrm{~m})$ & 42,52568700 & $-219,66062960$ \\
$\mathrm{~T}_{z}(\mathrm{~m})$ & 143,65810502 & 77,00212336 \\
$(1+\lambda)$ & 1,00000370 & 1,00000978 \\
$\mathrm{R}_{x}(\mathrm{rad})$ & 0,00000194 & 0,00003235 \\
$\mathrm{R}_{y}(\mathrm{rad})$ & $-0,00003652$ & $-0,00001017$ \\
$\mathrm{R}_{z}(\mathrm{rad})$ & 0,00001397 & $-0,00001476$ \\
\hline
\end{tabular}

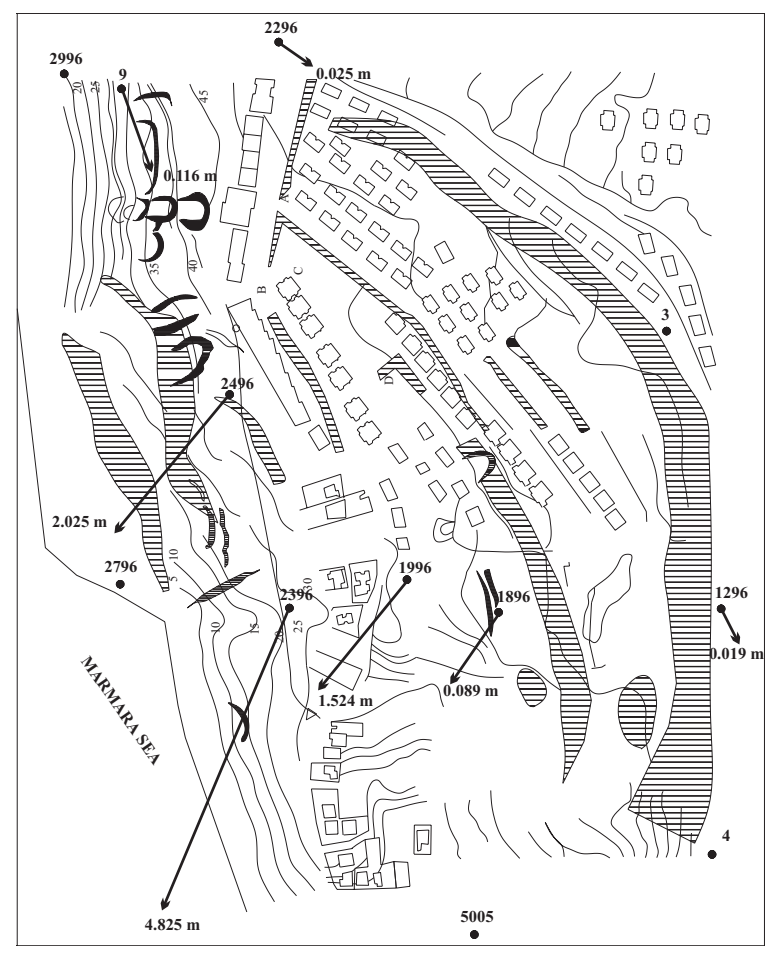

Fig. 1. Horizontal displacements obtained by GTLS method.

the weighting procedure introduced in the GTLS approach. In case the both coordinate sets have been assumed to be

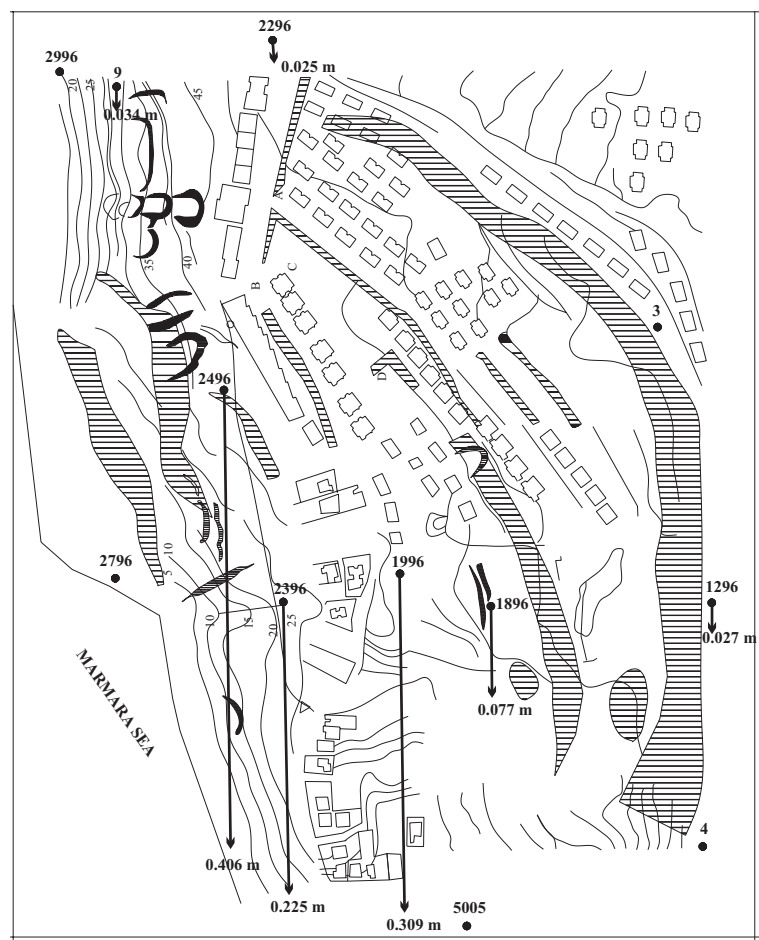

Fig. 2. Vertical displacements obtained by GTLS method.

equally weighted, namely the $\mathrm{D}=\mathrm{I}_{n \times n}$ and $\mathrm{C}=\mathrm{I}_{5 \times 5}$, two estimates will be closer.

Transformed coordinates obtained from both LS and GTLS solutions are given in Table 3. As expected, the transformed coordinates from the two methods are close to each other even though the parameters used for the transformations are significantly different, as mentioned earlier.

After the calculation of coordinates, a similarity deformation analysis explained in Sect. 4 has been applied using the results obtained by the GTLS solution. Figures 1 and 2 show horizontal and vertical point displacements obtained through GTLS method, respectively. In order to visualise, the deformation components have been presented in separate figures. The deformation vectors seen in Fig. 1 are drawn using plane coordinates. As seen from the figures, 
Table 3. Transformed coordinates by LS and GTLS solution.

\begin{tabular}{|c|c|c|c|c|c|c|}
\hline \multirow[t]{2}{*}{ Point ID } & \multicolumn{3}{|c|}{ Transformed Coordinates by LS } & \multicolumn{3}{|c|}{ Coordinate differences } \\
\hline & $X(m)$ & $\mathrm{Y}(\mathrm{m})$ & $\mathrm{Z}(\mathrm{m})$ & $\mathrm{dx}(\mathrm{m})$ & dy $(m)$ & $\mathrm{dz}(\mathrm{m})$ \\
\hline 4 & 4233377,6444 & 2308363,2638 & 4161191,9549 & 0.0090 & 0.0115 & 0.0072 \\
\hline 9 & 4233224,8160 & 2307823,5210 & 4161590,9233 & -0.0289 & 0.1014 & -0.0101 \\
\hline 1296 & 4233289,4809 & 2308332,2473 & 4161303,0185 & 0.0069 & 0.0158 & 0.0158 \\
\hline 1896 & 4233317,6968 & 2308237,1644 & 4161294,3658 & -0.0182 & 0.0692 & 0.0866 \\
\hline 1996 & 4233368,6083 & 2308086,8449 & 4161292,2962 & -0.9146 & 0.8801 & 0.8950 \\
\hline 2296 & 4233140,8996 & 2307879,7426 & 4161660,1989 & 0.0092 & 0.0073 & -0.0124 \\
\hline 2396 & 4233420,5926 & 2307995,1864 & 4161257,3776 & -3.1657 & 3.1995 & 1.7662 \\
\hline 2496 & 4233323,5753 & 2307939,6519 & 4161415,2642 & -1.2093 & 1.2024 & 1.1529 \\
\hline \multirow[t]{2}{*}{ Point Number } & \multicolumn{3}{|c|}{ Transformed Coordinates by GTLS } & \multicolumn{3}{|c|}{ Coordinate differences } \\
\hline & $\mathrm{X}(\mathrm{m})$ & $\mathrm{Y}(\mathrm{m})$ & $\mathrm{Z}(\mathrm{m})$ & $\mathrm{dx}(\mathrm{m})$ & dy $(m)$ & $\mathrm{dz}(\mathrm{m})$ \\
\hline 4 & 4233377,6379 & 2308363,2683 & 4161191,9435 & 0.0025 & 0.0160 & -0.0042 \\
\hline 9 & 4233224,8136 & 2307823,5300 & 4161590,9267 & -0.0313 & 0.1104 & -0.0067 \\
\hline 1296 & 4233289,4718 & 2308332,2525 & 4161303,0064 & -0.0022 & 0.0210 & 0.0037 \\
\hline 1896 & 4233317,6909 & 2308237,1696 & 4161294,3573 & -0.0241 & 0.0744 & 0.0781 \\
\hline 1996 & 4233368,6071 & 2308086,8506 & 4161292,2936 & -0.9158 & 0.8858 & 0.8924 \\
\hline 2296 & 4233140,8932 & 2307879,7517 & 4161660,1988 & 0.0028 & 0.0164 & -0.0125 \\
\hline 2396 & 4233420,5952 & 2307995,1920 & 4161257,3789 & -3.1631 & 3.2051 & 1.7675 \\
\hline 2496 & 4233323,5747 & 2307939,6591 & 4161415,2657 & -1.2099 & 1.2096 & 1.1544 \\
\hline
\end{tabular}

Table 4. Significance tests of the deformation points according to LS and GTLS results.

\begin{tabular}{lllll}
\hline Point ID & $\begin{array}{l}\text { Test value } \\
\text { LS }\end{array}$ & $\begin{array}{l}\text { Test value } \\
\text { GTLS }\end{array}$ & $\begin{array}{l}\text { Fractile value of } \\
\text { the test statistics }\end{array}$ & $\begin{array}{l}\text { Final Decision due } \\
\text { to LS / GTLS (Y or N) }\end{array}$ \\
\hline 4 & 0.612 & 0.435 & 2.609 & $\mathrm{~N} / \mathrm{N}$ \\
9 & 70508 & 80180 & & $\mathrm{Y} / \mathrm{Y}$ \\
1296 & 2.319 & 4.389 & $\mathrm{~N} / \mathrm{Y}$ \\
1896 & 56725 & 60796 & $\mathrm{Y} / \mathrm{Y}$ \\
1996 & 12333.867 & 12380.648 & $\mathrm{~N} / \mathrm{N}$ \\
2296 & 2.755 & 4.001 & $\mathrm{~N} / \mathrm{Y}$ \\
2396 & 109363.179 & 109438.712 & $\mathrm{Y} / \mathrm{Y}$ \\
2496 & 23535.388 & 23614.568 & $\mathrm{Y} / \mathrm{Y}$ \\
\hline
\end{tabular}

horizontal displacements up to $4.826 \mathrm{~m}$ and vertical downward displacements up to $0.406 \mathrm{~m}$ have been detected.

Table 4 summarises the significance tests for the deformation points regarding the both LS and GTLS methods. The test criterion is the fractile value of the t-distribution with 95\% confidence level and relevant degree of freedom of the problem. The deformed points are denoted by $\mathrm{Y}$ while the stable ones are indicated by N. Note that the points 1296 and 2296 were detected as deformed points through the GTLS method whereas they were detected as stable using classical LS approach. Since the difference between the fractile value and the test value of the point 2296 for LS approach is quite small, it is neglected by the software used for analysis. The GTLS method takes the covariances of the point coordinates into account and therefore produces more reliable values as the deformation vectors to be tested for significance.

\section{Conclusions}

The traditional techniques used for solving the linear estimation problems are based on classical LS. Even though some robust methods based on $\mathrm{L}_{1}$-norm do exist, either the LS or robust estimation techniques assume that only the observation vector contains errors. However, this assumption is not valid for every case. One example for such a problem is the coordinate transformation. Especially in deformation surveys, coordinates of the points of the monitoring network are 
computed independently using the set of observations held in individual surveying campaigns. Each surveying campaign is processed independently and usually a free network adjustment is carried out. Therefore, the adjusted coordinates of the points are obtained with their covariance matrices. Considering the coordinate transformation between two coordinate sets, one can easily see that the observations and the partly the design matrix of the transformation are erroneous. One solution to this problem is the application of TLS method for estimation of the transformation parameters. TLS method considers the erroneous design matrix as well as their covariance information in computations.

This study focuses on the use of TLS approach for geodetic deformation analysis. For comparison a traditional approach, namely similarity transformation has also been applied on the same data set. The big difference in the estimated parameters using LS and TLS (see Table 5) comes from the errors and the covariance of the coordinates of the points which were involved in the design matrix. However, the main part of the difference is due to the different covariance of the both system coordinates, which were employed as relative scaling between the observation vector and the erroneous columns of the design matrix. Thus, we can conclude that the transformation parameters of a Helmert transformation problem are strongly sensitive to the accuracy of the coordinates of the identical points.

Recalling the transformed coordinates of the object points given in Table 6, the differences between the LS and TLS solution are in cm levels. Though, this level of difference is not very important for such a study area where there are great displacements, such level of difference may play an important role in monitoring of big engineering structures (e.g., reservoir dams) where very small displacements are critical.

Finally, we propose TLS estimation technique for use in geodetic deformation analysis studies in order to reduce the uncertainty in the classical LS model and to obtain more realistic information about the deformation or displacement vectors which are to be tested statistically. As a future work, we will apply other methods (e.g., Total Least Norm method) for EIV models. In addition to $\mathrm{L}_{2}$-norm estimations, we will compose a robust EIV estimation model based on the minimisation of the error in discrete $\mathrm{L}_{1}$-norm so that the deformation analysis will be held during the transformation process without any further statistical tests.

Acknowledgements. The authors would like to thank to the Geodetic Institute, University of Karlsruhe that they have given CODEKA3D deformation analysis software for scientific use in our institute. And also as the partner of the deformation measurements project of Gürpinar, the Institute of Physical Geodesy, Darmstadt University of Technology are gratefully acknowledged.

Edited by: S. Tinti

Reviewed by: two referees

\section{References}

Akyilmaz, O.: Total Least Squares solution of coordinate transformation, to be published in Survey Review, in press, 2005.

Ayan, T.: Adjustment computations, Lecture Notes, Istanbul Technical University, Istanbul, Turkey, in Turkish, 85, 2001.

Ayan, T.: Mathematical statistics and hypothesis testing, Postgraduate Lecture Notes, Istanbul Technical University, Istanbul, Turkey, in Turkish, 101, 1981.

Caspary, W. F.: Concept of network analyses, edited by: Rüger, J. M., School of Surveying, University of New South Wales, New South Wales, Australia, 25, 1987.

De Groen, P.: An introduction to Total Least Squares, Nieuw Archief voor Wiskunde, 14, 237-253, 1996.

Denli, H. H.: Crustal Deformation Analysis in the Marmara Sea Region, J. Surveying Engineering, 130(4), 151-155, 2004.

Felus, Y.: Application of Total Least Squares for spatial point process analysis, J. Surveying Engineering, 130(3), 126-133, 2004.

Golub, H. G. and Van Loan, F. C.: An analysis of the Total Least Squares problem, SIAM J. Numerical Analysis, 17(6), 883-893, 1980.

Koch, K. R.: Parameter estimation and hypothesis testing in linear models, 2nd Ed., Springer, Berlin, 249, 1999.

Leon, S.: Linear algebra with applications, 6th Ed., Prentice Hall, Upper saddle River, N. J., 202-203, 2002.

Markovsky, I., Van Huffel, S., and Kukush, A.: On the computation of the multivariate structured Total Least Squares estimator, $\mathrm{Nu}$ merical Linear Algebra with Applications, 11, 591-608, 2004.

Mikhail, E. M. and Ackermann, F.: Observations and least squares, Harper \& Row publishers, New York, 128, 1976.

Mitsakaki, C.: Coordinate transformations, in: Proceedings of FIG Working Week 2004, Athens, Greece, 22-27 May 2004.

Mühlich, M. and Mester, R.: Unbiased errors-in-variables estimation using generalized eigensystem analysis, in: Statistical Methods in Video Processing, edited by: Comaniciu, D., Kanatani, K.-I., Mester, R., Suter, D., ECCV 2004 Workshop SMVP 2004, Prague, Czech Republic, 16 May 2004, Revised Selected Papers, Lecture Notes in Computer Science 3247, Springer, 38-49, 2004.

Lenzmann, L. and Lenzmann, E.: Rigorous adjustment of the nonlinear Gauss-Helmert Model, Allgemeine VermessungsNachrichten, 111(2), 68-73, Wichmann-Huethig, (in German), 2004.

Van Huffel, S.: The generalized Total Least Squares problem: formulation, algorithm and properties, in: Numerical Linear Algebra, Digital Signal Processing and Parallel Algorithms, edited by: Golub, G. H. and Dooren, P. V., NATO ASI, Series F, No. 70, Springer, Berlin, 651-660, 1991.

Van Huffel, S. and Vandewalle, J.: The Total Least Squares problem: computational aspects and analysis, Society for Industrial and Applied Mathematics (SIAM), 47, 1991.

Wolf, R. P. and Ghilani, D. C.: Adjustment computations: statistics and least squares in surveying and GIS, Wiley, New York, 347, 1997. 